\title{
Impact of surface wettability on S-layer recrystallization: a real-time characterization by QCM-D
}

\author{
Jagoba Iturri ${ }^{* 1}$, Ana C. Vianna ${ }^{1,2}$, Alberto Moreno-Cencerrado ${ }^{1}$, Dietmar Pum ${ }^{1}$, \\ Uwe B. Sleytr ${ }^{1}$ and José Luis Toca-Herrera ${ }^{* 1}$
}

\author{
Full Research Paper \\ Address: \\ ${ }^{1}$ Institute for Biophysics, Dept. of Nanobiotechnology, BOKU \\ University for Natural Resources and Life Sciences, Muthgasse 11 \\ (Simon Zeisel Haus), A-1190 Vienna, Austria and 2University of Sao \\ Paulo (USP), Faculty of Philosophy, Science and Letters of Ribeirao \\ Preto (FFCLRP), Department of Chemistry, Ribeirao Preto, SP, Brazil \\ Email: \\ Jagoba Iturri* - jagoba.iturri@boku.ac.at; José Luis Toca-Herrera* - \\ jose.toca-herrera@boku.ac.at \\ * Corresponding author \\ Keywords: \\ bacterial S-layers; Quartz crystal microbalance with dissipation \\ monitoring (QCM-D); recrystallization kinetics; surface wettability
}

Beilstein J. Nanotechnol. 2017, 8, 91-98. doi:10.3762/bjnano.8.10

Received: 05 September 2016

Accepted: 21 December 2016

Published: 11 January 2017

Associate Editor: D. J. Müller

(c) 2017 Iturri et al.; licensee Beilstein-Institut. License and terms: see end of document.

\begin{abstract}
Quartz crystal microbalance with dissipation monitoring (QCM-D) has been employed to study the assembly and recrystallization kinetics of isolated SbpA bacterial surface proteins onto silicon dioxide substrates of different surface wettability. Surface modification by UV/ozone oxidation or by vapor deposition of $1 \mathrm{H}, 1 \mathrm{H}, 2 \mathrm{H}, 2 \mathrm{H}$-perfluorododecyltrichlorosilane yielded hydrophilic or hydrophobic samples, respectively. Time evolution of frequency and dissipation factors, either individually or combined as the so-called $D f$ plots, showed a much faster formation of crystalline coatings for hydrophobic samples, characterized by a phase-transition peak at around the $70 \%$ of the total mass adsorbed. This behavior has been proven to mimic, both in terms of kinetics and film assembly steps, the recrystallization taking place on an underlying secondary cell-wall polymer (SCWP) as found in bacteria. Complementary atomic force microscopy (AFM) experiments corroborate these findings and reveal the impact on the final structure achieved.
\end{abstract}

\section{Introduction}

Crystalline bacterial protein layers (S-layers) are arrays of (glyco)proteins $\left(M_{\mathrm{w}}\right.$ of 40 to $200 \mathrm{kDa}$ ) forming the outermost envelope of prokaryotes, and represent the simplest biological membrane [1,2]. Depending on the number of identical protein subunits forming each morphological unit, S-layers show oblique (p1, p2), square (p4), or hexagonal (p3, p6) lattice symmetries, with unit cell dimensions ranging from 3 to $30 \mathrm{~nm}$ [3]. More specifically, the S-layer protein employed in this work, the SbpA from Lysinibacillus sphaericus CCM2177, presents a square (p4) lattice symmetry with a reported spacing of around $13 \mathrm{~nm}$ and base angle of $90^{\circ}$ in its natural environment [4].

From the point of view of bioengineering, it is highly interesting that isolated S-layer protein subunits have the ability to selfassemble, through the so-called recrystallization process, on a 
broad number of supports (see the general scheme in Figure 1). In former studies, the capability of SbpA to recrystallize from bulk has been tested on common bare substrates (silicon wafers, mica, gold) [5-9], with coatings of different nature (lipid bilayers, SAMs, polyelectrolyte multilayers) [10-14] and even on structures with an important contribution in the $Z$-axis, as recently reported for charged polyelectrolyte brushes [15]. However, some fundamental questions are yet to be solved about the S-layer recrystallization process under different physico-chemical conditions of the substrate (i.e., wettability or the presence of specific chemical groups and receptors), such as the different adsorption kinetics, crystal domain sizes and lattice symmetry parameters obtained. The importance of such factors for the morphology and biological function of isolated proteins has been already shown in literature [16].

In this regard, the quartz crystal microbalance with dissipation monitoring (QCM-D) has proven to be a powerful technique to follow in situ the binding of S-layers on different supports $[17,18]$. The QCM-D responses, i.e., the resonance frequency $f$ and the energy dissipation $D$ of the shear oscillatory motion of a piezoelectric quartz crystal sensor, change upon adsorption or desorption of material on the surface of that sensor. The measured parameters are highly sensitive to the mass (of the order of a few nanograms per square centimeter) and the mechanical properties of the surface-bound layer and can be monitored simultaneously and in real time. Conceptually, differences in frequency reflect interaction of the bacterial protein with the substrate, while dissipation changes are related to the structural rearrangement and variation of the viscous contribution of the film. The combination of both factors in the so-called $D f$ plots ( $\Delta D$ vs $\Delta f$ ) enhances the information extracted, in comparison to the individual measurements, and has become a very useful tool to characterize the formation of biofilms in the recent years [19-23].

In this manuscript, our study is focused on a physico-chemical analysis about how changes in the wettability (hydrophilic vs hydrophobic) of $\mathrm{SiO}_{2}$ surfaces influence the recrystallization pathways of SbpA proteins. By means of either oxidative treatments (UV/ozone) or vapor deposition of a fluorinated silane the wetting properties of the substrate could be easily tailored to be hydrophilic or hydrophobic. Then, exposure of the samples to $\mathrm{SbpA}$ and their subsequent evolution in time was followed in situ by QCM-D with the support of atomic force microscopy for topographical analysis of the resulting crystal-like films. The results are compared with the biomimetic case found in bacteria represented by the interaction of SbpA with a secondary cellwall polymer (SCWP), which specifically recognizes the N-terminal region of the S-layer protein $[24,25]$.

\section{Results and Discussion \\ S-layer formation on secondary cell-wall polymer (SCWP) films}

Both the binding of thiolated-SCWP to gold surfaces and its subsequent use as underlying material for the recrystallization of SbpA bacterial protein could be monitored in real time by means of QCM-D (Figure 2). As the first step, the in vitro formation of a SCWP film mimicking the underlying material found by bacterial S-layer proteins in nature (Figure 2a) induced a variation in frequency of $30 \mathrm{~Hz}$, corresponding to a bound mass of about $0.53 \mu \mathrm{g} / \mathrm{cm}^{2}$ (Figure $2 \mathrm{~b}$ ). The low values measured for $\Delta D$ (below $1 \times 10^{-6}$ ) hint at a very thin film that can approximately be calculated to be about 5-6 $\mathrm{nm}$, and at a low water content of the film. This result agrees with what is expected from the internal arrangement of the polymer [25].

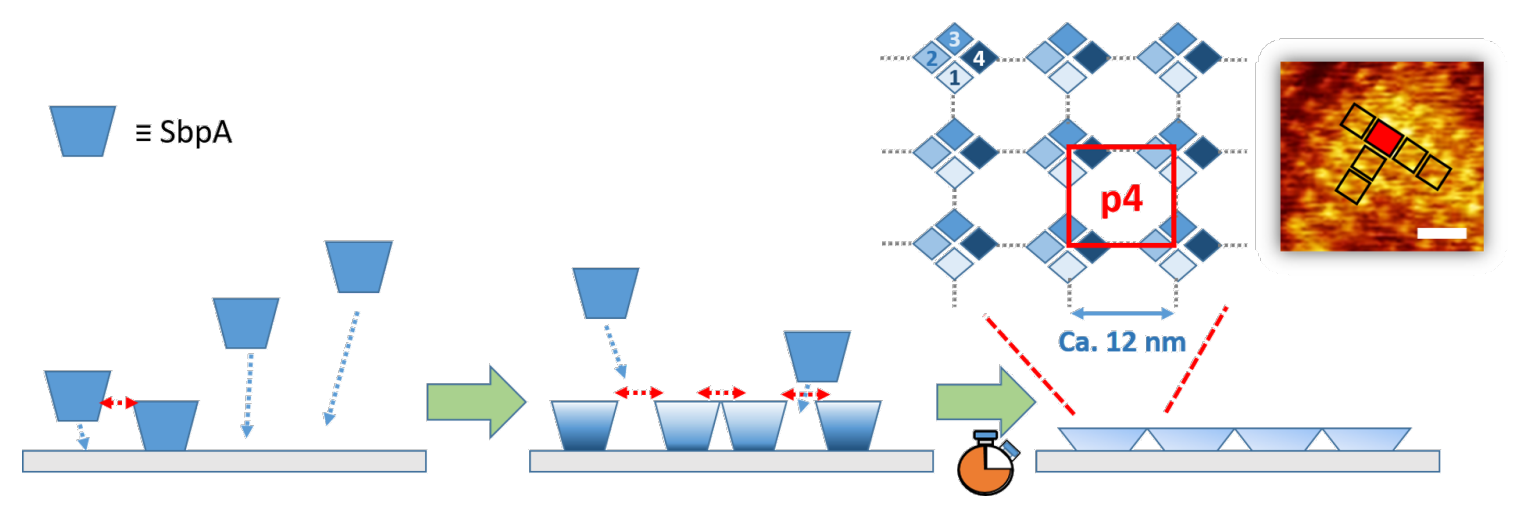

Figure 1: General scheme of SbpA protein S-layer crystal formation on a planar substrate: (left) the initial approach and binding of protein units to the surface co-exists with inter-monomeric interactions that lead to the formation of first nucleation points. Incorporation of additional building blocks takes place in parallel to the first crystal formation (center), which regulates the SbpA-film interacting capability throughout the process. After a certain incubation time, completion of the protein assembly leads to a full coverage of the surface (right). This film appears as a crystalline coating with regular square-like ( $\mathrm{p} 4$ ) lattice, with four SbpA sub-units involved, as shown in the AFM height image. The scale bar corresponds to $25 \mathrm{~nm}$. 


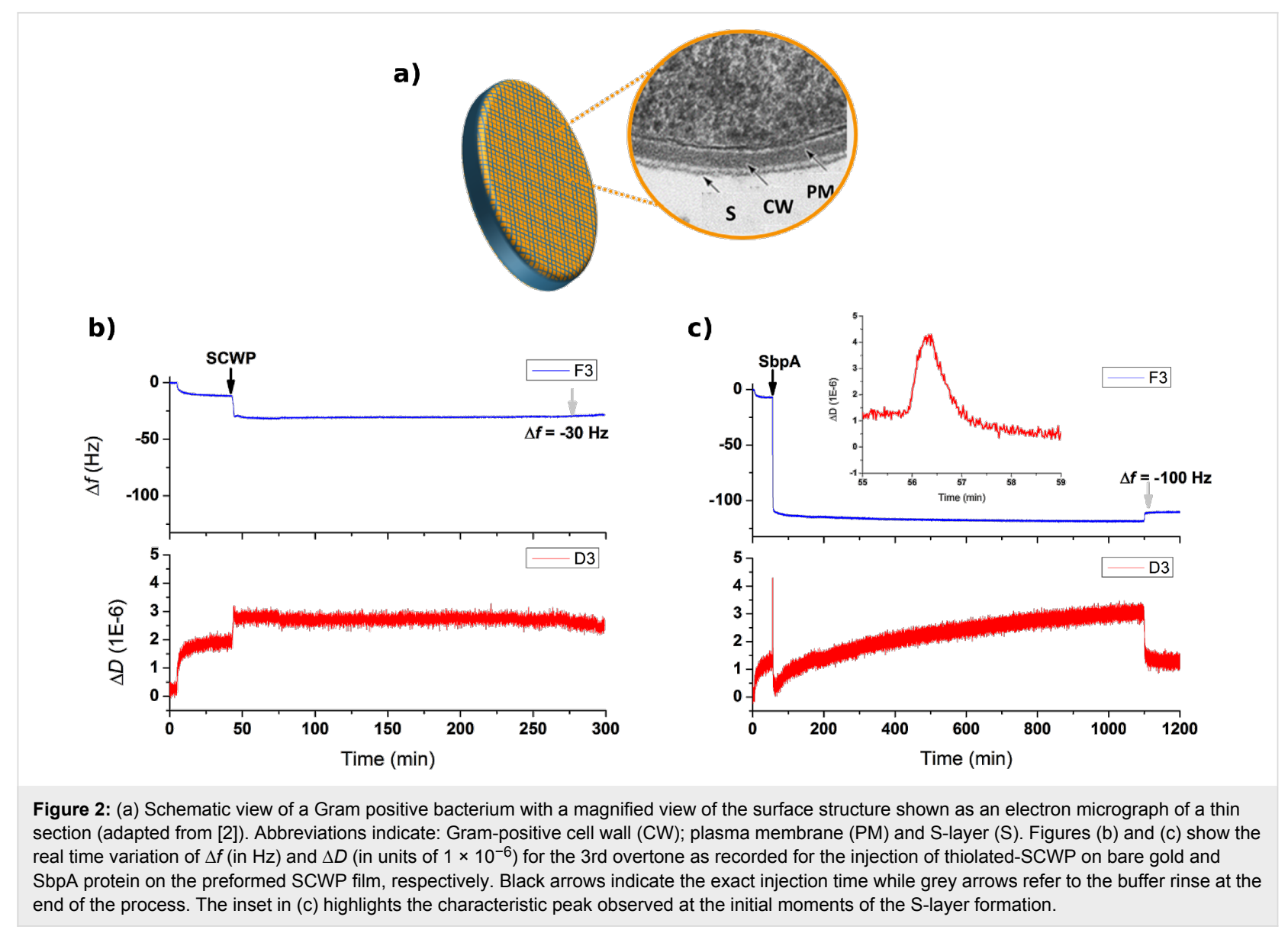

However, this slight modification is sufficient to provide a new character to the sensor.

Subsequently, the SCWP was exposed to a $0.1 \mathrm{mg} / \mathrm{mL}$ solution of SbpA in crystallization buffer (CB) and left to evolve overnight. As shown in Figure 2c this protein injection caused a sudden decrease in $\Delta f$ down to values of around $-100 \mathrm{~Hz}$. After that a plateau that remained throughout the incubation time (ca. $17 \mathrm{~h}$ ) was reached quickly. The steadiness of the values derives from null mass adsorption over this period or, in other words, the full completion of the coating, which prevents further binding of SbpA units from the bulk. Final rinse with $\mathrm{CB}$ is employed to quench the incubation, causing no remarkable changes in the S-layer structure besides the flushing of unbound protein residues. The related variation in dissipation provides complementary information about the film formation process, since the drop in frequency is accompanied simultaneously by an increase in $\Delta D$ which, after a maximum, drops rapidly to much lower values. This peak appearance can be explained by a transitional step from mere protein adsorption (inducing an increase in $\Delta D$ ) to a sudden stiffening of the film as SbpA keeps attaching. This behavior can be explained by the simultaneously ongoing recrystallization. Then, dissipation in- creases slightly back for the next 100 min and subsequently maintains a linear growth, with extremely low steepness, until $\Delta D=3 \times 10^{-6}$. The final rinse with buffer caused the drop of that value to a half.

A better way to follow the concomitant evolution of the protein layer in terms of bound mass and viscoelasticity of the film is by means of the so-called $D f$ plots, as seen in Figure 3. It must be noted that the starting coordinates $(0,0)$ were adapted to exactly refer to the moment at which SbpA is injected into the chamber while the red-to-blue color variation reflects the elapsed time, as denoted by the color scale on the inset. Thus, Figure 3a shows the increasing trend of $\Delta D$, up to a maximum value $\left(3.5 \times 10^{-6}\right)$ is only observed for the initial variation of 50-60 Hz. After this maximum value, the subsequent SbpA binding is followed by an almost linear drop in $\Delta D$ down to values even below the starting value. This is only possible because of the softness of the layer underneath, which might collapse slightly upon S-layer deposition on top. At a frequency variation of $-100 \mathrm{~Hz}$, the process seems to reach a second stage after which no additional protein is bound, but only $\Delta D$ undergoes a change. The vertically increasing dissipation might correspond to a slow system rearrangement caused by the si- 
a)

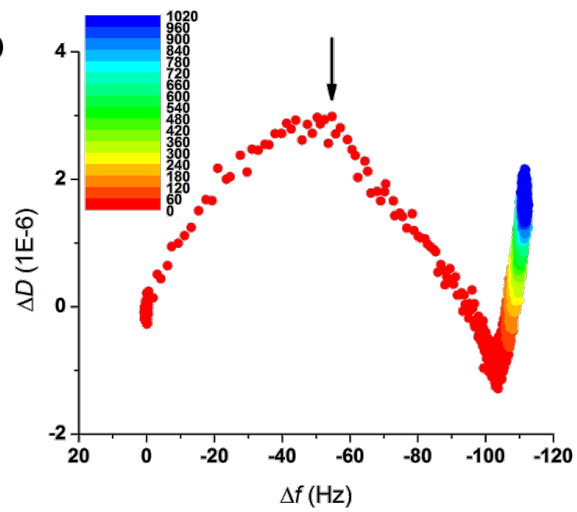

b)

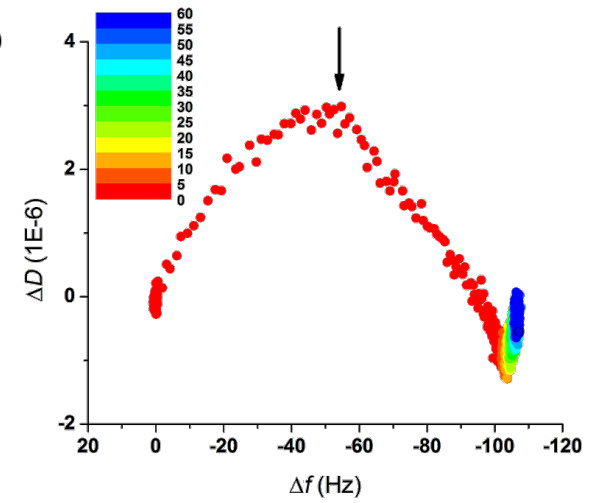

Figure 3: $D f$ plots representing the binding of SbpA onto the SCWP film. The color scales indicate the elapsed time after protein injection for (a) the full incubation process (1020 $\mathrm{min}$, color step $60 \mathrm{~min}$ ) and (b) the initial $60 \mathrm{~min}$ of the process (5 min color steps). Black arrows indicate the transition point towards crystalline state.

multaneous motility of the supporting SCWP film and the lateral reorganization of the S-layer, both leading to the final structure. While both the film formation and recrystallizationinduced hardening take place in the initial $60 \mathrm{~min}$ of the incubation, it takes around $16 \mathrm{~h}$ for the gradual film adjustment. Indeed, as shown in Figure 3b, the S-layer formation can be assumed to be almost completed within the first $15 \mathrm{~min}$. These results regarding $\mathrm{SbpA}$ recrystallization on a nature-mimicking SCWP film will be considered for comparative and descriptive purposes in the following section.

\section{S-layer formation on hydrophobic and hydrophilic $\mathrm{SiO}_{2}$}

In order to investigate the impact that surface wettability has on the binding and recrystallization of SbpA in the absence of specific protein-substrate interactions, the above mentioned protocol was again followed for silicon dioxide substrates under different wettability conditions: either UV/ozone-treated (hydrophilic, water contact angle $\Theta<10^{\circ}$ ) or vapor-coated with a fluorosilane $\left(\Theta=90^{\circ}\right)$. Figure $4 \mathrm{a}$ depicts the time evolution of both $\Delta f$ and $\Delta D$ upon injection of $0.1 \mathrm{mg} / \mathrm{mL} \mathrm{SbpA} \mathrm{in} \mathrm{CB.} \mathrm{It}$ can be observed how from the very initial moments of the incubation, the behavior of SbpA is different on hydrophilic $\mathrm{SiO}_{2}$ substrates compared to that on hydrophobic fluorinated $\mathrm{SiO}_{2}$. A frequency plateau is reached in the former case after a decrease of only $-60 \mathrm{~Hz}$, while in the latter case it drops down to $-100 \mathrm{~Hz}$ before stabilization takes place. In addition, recrystallization on the hydrophobic substrate is accompanied by the characteristic peak in dissipation but does not occur for the binding of SbpA to a hydrophilic sample. After $3 \mathrm{~h}$ incubation and attending to unvarying $\Delta f$ and $\Delta D$ values, especially in the fluorinated substrate, the process was stopped by flushing of
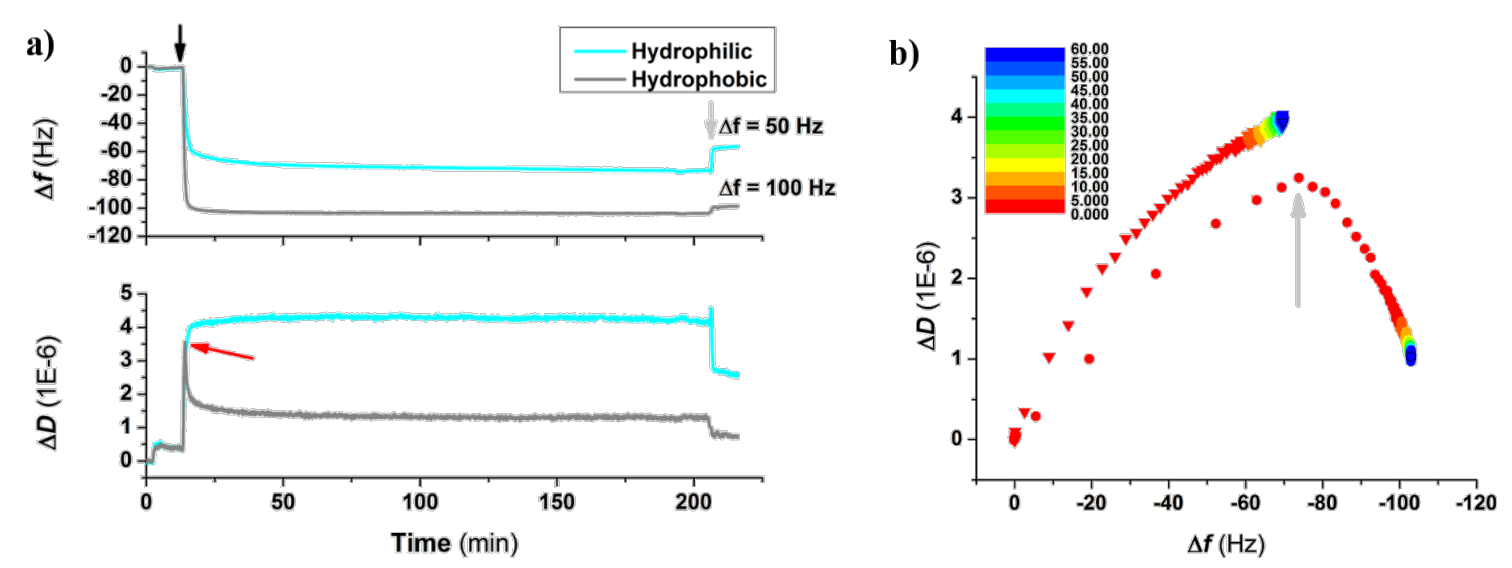

Figure 4: (a) Time evolution of $\Delta f$ and $\Delta D$ for the injection of SbpA protein on both hydrophilic and hydrophobic $\mathrm{SiO}_{2}$ substrates. The black arrow indicates the exact moment of protein injection while the grey arrows refer to the buffer rinse at the end of the process. The red arrow points out the appearance of the peak in $\Delta D$ in the hydrophobic case. In (b), the $D f$ plot comparing the binding of SbpA onto both substrates is shown for the initial $60 \mathrm{~min}$ of the process, as indicated by the color scale. Grey arrow indicates the transition point. 
CB. It is already well known by the authors that long incubation $(>15 \mathrm{~h})$ of SbpA on hydrophilic substrates leads to the formation of a crystal-like film. However, because of the lack of resemblance to the natural case (in bacteria), these results were not considered for further comparison. However, similar results to those of the hydrophobic substrate in terms of frequency variation, as well as the presence of the structural transition peak in $\Delta D$, have already been reported in previous studies as developed by our group for different types of hydrophobic alkylsilanes (octadecyltrichlorosilane) [17] and alkylthiols (dodecanethiol and hexanethiol) [18] on silica and gold, respectively. In those cases the real-time analysis differed significantly from the one carried out here.

Figure $4 \mathrm{~b}$ shows the corresponding $D f$ plot for the initial $60 \mathrm{~min}$ of the two processes described above and indicates the surface wettability-dependent trend. SbpA binding on the hydrophobic surface repeats the 2-step formation seen for SCWP, with a clear transition peak taking place. In contrast, the binding to hydrophilic $\mathrm{SiO}_{2}$ shows a much slower process, with no characteristic transition peak in it, and what seems to be a still ongoing dissipation increase.

\section{Comparison between SCWP and hydrophobic $\mathrm{SiO}_{2}$}

A quick glimpse at the QCM-D results discussed above shows the high similarity between the recrystallization processes on both the nature-mimicking SCWP and the hydrophobic $\mathrm{SiO}_{2}$. This is a remarkable outcome: The natural case is featured by, among others, specific protein-carbohydrate interactions involved in the S-layer formation [25], while on fluorinated$\mathrm{SiO}_{2}$ the protein binding and crystal formation is mainly driven by hydrophobic interactions (non-specific). Also, SCWP shows a rather hydrophilic behavior (contact angle $\Theta<10^{\circ}$ ), opposite to that of the fluorosilane. However, and in addition to the transition peak shown in $\Delta D$ and the final SbpA mass bound, the rapidity by which the S-layer formation process reaches completion on both surfaces is practically the same.

In Figure 5a the evolution of the recrystallization process for the initial $10 \mathrm{~min}$ is compared for both using the respective $D f$ plots. As can be observed, besides the fact that both, the trend followed and the absolute values reached (including the same times required for formation and the $\Delta D$ maximum values at the transition peak), are certainly comparable. However, a deeper analysis highlights the existence of a few differences: Firstly, the position at which the transition towards a higher rigidness takes place is shifted in frequency. This means that in the case of the SCWP a lower amount of mass needs to be bound (55\% of the total mass). For fluorinated substrates almost the $75 \%$ of the mass is required before the observation of the characteristic peak. Secondly, the capability of S-layers to rearrange after complete SbpA deposition changes from one system to the other. As mentioned above, the S-layer on top of the SCWP seems to have the ability of undergoing a rearrangement, which is reflected by a gradually increasing dissipation as the system is left to evolve. Such behavior already appears within the first $60 \mathrm{~min}$ of incubation, as indicated by the final slope in Figure $3 b$. On the contrary, recrystallization on hydrophobic $\mathrm{SiO}_{2}$ shows no trace of final rearrangement after the films is formed.

The most plausible explanation for these points relies on two factors, namely, the motility of the underlying layer and that of the protein film, which regulate the capability of the system to minimize the energy and lead to the mentioned differences in the final layout. For a fluorosilane coating underneath, the standing positions of the S-layer are somehow fixed and the lateral motion of the film is restricted. This is completely opposite in the case of a SCWP-coated substrate. This is probably better understood during long incubations, but also seems to play a key role in the very first moments. A good example of a)

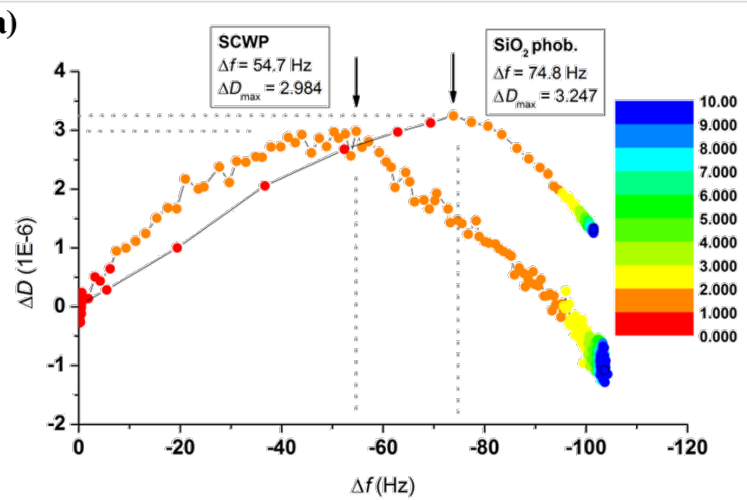

b)

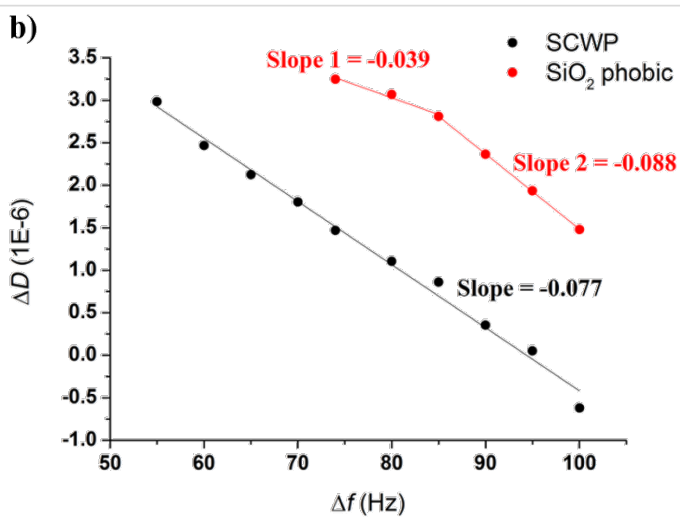

Figure 5: (a) $D f$ plot comparing the binding of SbpA onto SCWP and hydrophobic $\mathrm{SiO}_{2}$ for the initial 10 min. (b) $D f$ slope evolution after achievement of the maximum value of $\Delta D$. 
such influence can be seen in Figure 5b. The slope measured immediately after the transition peak in SCWP is twice of that of $\mathrm{SiO}_{2}$, and stays constant along the rest of the process, while the slope in the latter only drops for the last $15 \%$ of the S-layer formation. Although the SbpA adsorption process requires only about $2 \mathrm{~min}$ in both examples, the substrate motility leads to achievement of lower $\triangle D$ values on SCWP because of this concomitant re-organization as SbpA attaches, in addition to the specific SbpA-SCWP interactions. As a result, also the number of SbpA units required to start the transition towards crystal formation decreases, as the process (i.e., the protein-protein contact) is favored by the polymer below.

In terms of the final topography, the atomic force micrographs shown in Figure 6 confirm what was deduced from the QCM-D results. The S-layer on top of the SCWP appears as an even coating consisting of a boundary-free crystalline domain (Figure 6a), in contrast to the multiple small domains forming the S-layer on fluorosilane with visible physical boundaries between them (Figure 6b). The included fast Fourier transform (FFT) images contribute to visualize the uniformity of the crystalline layers. For SCWP the FFT appears as a well-defined square, resulting from a unique crystal orientation while the halo observed for hydrophobic substrates derives from the multiple crystal orientations. In both cases the measured lattice spacing was $13 \mathrm{~nm}$ from center to center. Therefore, the continuous re-organization of the crystalline film as it is formed, allowed by the motility of the supporting polymer below, leads to a very homogeneous S-layer, of high resemblance to the one found on bacterial surfaces [1].

\section{Conclusion}

S-layer recrystallization of SbpA bacterial protein has been shown to be highly influenced by surface wettability of the sup- porting substrate. Characterization of the recrystallization process on top of both hydrophilic and fluorosilane-modified hydrophobic $\mathrm{SiO}_{2}$ surfaces led to large differences in terms of process kinetics and development, as observed from the respective real-time physicochemical analysis. S-layer membrane formation on a hydrophobic sample resembled more faithfully the behavior of nature-mimicking secondary cell-wall polymer films, including the intermediate transition stages, besides the existing differences between both substrates. However, the fluid character of the underlying SCWP has a strong impact on the lateral motility as SbpA binds and enables a different final arrangement of the coating.

\section{Experimental \\ Materials}

Bacterial cell-surface layer protein, $\operatorname{SbpA}\left(M_{\mathrm{w}}=120 \mathrm{kDa}\right)$ was isolated and purified from L. Sphaericus CCM 2177 following the standard protocols [26]. Protein recrystallization buffer was prepared with $0.5 \mathrm{mM}$ Trizma base (Sigma, Germany) and $10 \mathrm{mM} \mathrm{CaCl}_{2}$ (98\% Sigma-Aldrich, Germany) and adjusted to $\mathrm{pH} 9$.

\section{Sample preparation}

S-protein preparation: After isolation, the protein solution was centrifuged at $5000 \mathrm{rpm}$ for $5 \mathrm{~min}$ in order to separate the S-protein monomers from self-assembly products and was stored at $4{ }^{\circ} \mathrm{C}$. Then, before each experiment, the supernatant solution was diluted using the appropriate amount of recrystallization buffer to a final concentration of $0.1 \mathrm{mg} / \mathrm{mL}$.

Assembly of fluorinated silane: Experiments were performed either ex situ onto silicon wafers or in situ onto QSX303 $\mathrm{SiO}_{2-}$ coated quartz sensors (4.95 MHz, Q-sense AB, Sweden). Both substrates were pre-treated with UV/ozone (BioForce a)

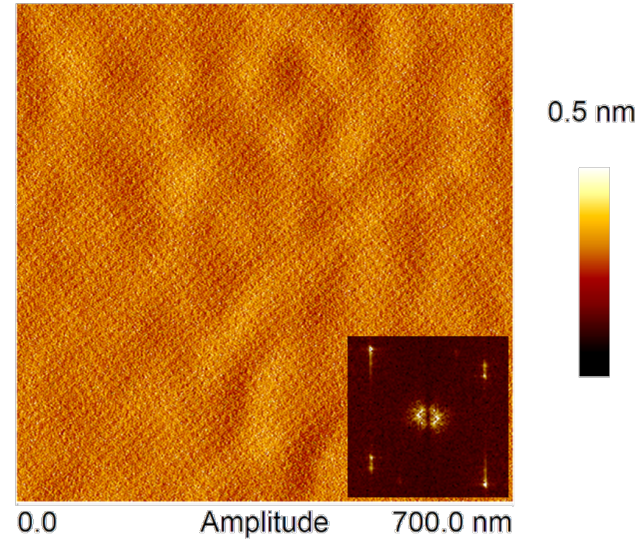

b)

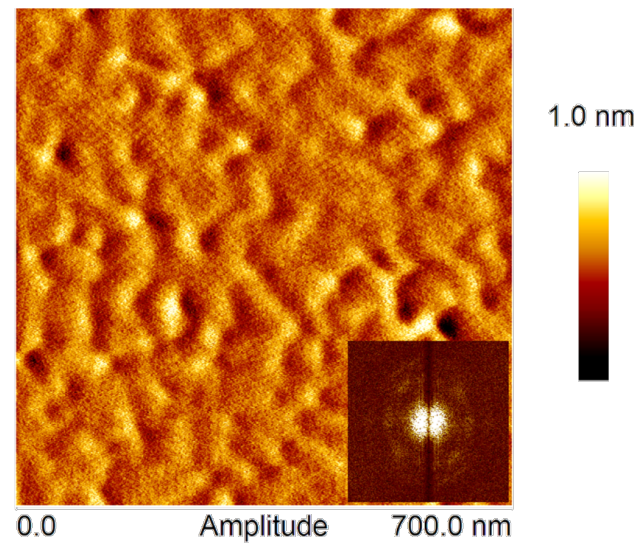

Figure 6: Atomic force amplitude micrographs $\left(700 \times 700 \mathrm{~nm}^{2}\right)$ showing the surface topography of SbpA recrystallized on (a) secondary cell-wall polymer and (b) fluorosilane-modified hydrophobic $\mathrm{SiO}_{2}$. Bottom insets show the fast Fourier transform images of the crystalline structures formed. 
Nanosciences, Ames, IA, USA) for $30 \mathrm{~min}$ and then placed overnight in an evacuated desiccator with ca. $30 \mu \mathrm{L}$ of $1 \mathrm{H}, 1 \mathrm{H}, 2 \mathrm{H}, 2 \mathrm{H}$-perfluorododecyltrichlorosilane (Sigma Aldrich) in chloroform for vapor silanization. Finally, they were rinsed with ethanol and water, dried under $\mathrm{N}_{2}$ and immediately used in the next step.

Secondary cell-wall polymer (SCWP) preparation: SCWP was isolated from peptidoglycan-containing sacculi of L. sphaericus CCM 2177 and purified [24]. Chemical modification of the reducing end on the polymer chains and introduction of a terminal sulfhydryl group by modification with 2-iminothiolane was performed [3]. Subsequently, thiol-secondary cellwall polymer (thiol-SWCP) was used to activate gold surfaces, either QSX301 gold sensors (4.95 MHz, QSense AB, Sweden) by means of peristaltic pumping (SM935C, Ismatec, Zürich, Switzerland), in QCM-D, or commercial flat gold Arrandee ${ }^{\mathrm{TM}}$ chips (Arrandee Metal GmbH, Germany) by immersion into a petri-dish filled with thiol-SCWP solution for AFM measuring purposes. After one hour of incubation we obtained a SCWP surface for S-protein recrystallization.

\section{Methods}

Atomic force microscopy (AFM): Recrystallized proteins were visualized using a multimode-AFM (Bruker AXS, Santa Barbara, USA) controlled by a Nanoscope V equipped with a J-scanner. Silicon-nitride probes (DNP-S, Bruker, USA) with a spring constant of about $0.3 \mathrm{~N} / \mathrm{m}$ were used in the experiments, which were calibrated on $\mathrm{SiO}_{2}$ using the thermal method. Prior to its use in the AFM fluid cell, the cantilever was cleaned with UV/ozone for $20 \mathrm{~min}$. Once mounted, the system was kept immersed in ultrapure water until stabilization of the deflection signal. Data acquisition was carried out in tapping mode, in order to not disturb the structure and at a scan rate lower than $2 \mathrm{~Hz}$.

The solutions containing recrystallization buffer and SbpA were injected into a chamber sealed by a silicone O-ring with a syringe. Before use, the fluid cell, tubing, and O-rings were washed overnight with $2 \%$ SDS, rinsed gently with ultrapure water, and dried with nitrogen. All images were processed with the Nanoscope program.

Quartz crystal microbalance with dissipation (QCM-D): QCM-D experiments were performed in a Q-Sense E4 instrument (Q-Sense AB, Sweden). Prior to their use, gold-coated quartz sensors (for thiol-SCWP binding) were sonicated in $2 \%(\mathrm{w} / \mathrm{w})$ SDS solution for $20 \mathrm{~min}$ and then rinsed with ultrapure water and ethanol. The crystals were dried under $\mathrm{N}_{2}$ stream, treated with UV/ozone for $30 \mathrm{~min}$ and mounted into the QCM-D chamber. For measurements involving the use of hydrophobic $\mathrm{SiO}_{2}$ sensors, these were treated as explained above. The same protocol was applied to hydrophilic $\mathrm{SiO}_{2}$ sensors with the exception of the overnight vapor deposition.

All experiments were performed at $25^{\circ} \mathrm{C}$. Real-time variations of Frequency $(\Delta f)$ and dissipation $(\Delta D)$ parameters were observed at several overtones $(n=3,5,7, \ldots, 13)$ throughout the QCM-D experiment. Additionally, the $\Delta D$-versus- $\Delta f$ plot can also be used to analyze QCM-D data. Each point of a $\Delta D-\Delta f$ plot represents a dissipation and frequency date at a certain time that provides a more detailed view on the viscoelastic evolution of films per mass unit $(\Delta m)$ change. The shape of the $\Delta D-\Delta f$ plot, as well as the slopes derived, can characterize specifically one type of process and enables the differentiation between them.

Contact angle measurements: Sessile-drop experiments were performed with a contact-angle measuring system (Kruss D100, Hamburg, Germany). Millipore water $(18.2 \mathrm{M} \Omega \cdot \mathrm{cm})$ was used as the liquid phase. Water drops (ca. $10 \mu \mathrm{L}$ ) were deposited on the substrates and the contact angles were obtained from the drop profiles.

\section{Acknowledgements}

Part of this work was supported by the International Graduate School BioNanoTech (IGS) Program of the Federal Ministry for Science and Research, Austria, and the Air Force Office of Scientific Research (AFOSR) (Grant FA9550-12-1-0274). A. C. Vianna also acknowledges the International Association for the Exchange of Students for Technical Experience (IAESTE) Programm.

\section{References}

1. Pum, D.; Toca-Herrera, J. L.; Sleytr, U. B. Int. J. Mol. Sci. 2013, 14, 2484-2501. doi:10.3390/ijms14022484

2. Sleytr, U. B.; Beveridge, T. J. Trends Microbiol. 1999, 7, 253-260. doi:10.1016/S0966-842X(99)01513-9

3. Sleytr, U. B.; Sára, M.; Pum, D.; Schuster, B. Prog. Surf. Sci. 2001, 68, 231-278. doi:10.1016/S0079-6816(01)00008-9

4. Sleytr, U. B.; Messner, P.; Pum, D.; Sára, M. Angew. Chem., Int. Ed. 1999, 38, 1034-1054.

doi:10.1002/(SICI)1521-3773(19990419)38:8<1034::AID-ANIE1034>3. $0 . C O ; 2-\#$

5. Györvari, E. S.; Stein, O.; Pum, D.; Sleytr, U. B. J. Microsc. 2003, 212, 300-306. doi:10.1111/j.1365-2818.2003.01270.x

6. Martin-Molina, A.; Moreno-Flores, S.; Perez, E.; Pum, D.; Sleytr, U. B.; Toca-Herrera, J. L. Biophys. J. 2006, 90, 1821-1829. doi:10.1529/biophysj.105.067041

7. Picher, M. M.; Küpcü, S.; Huang, C.-J.; Dostalek, J.; Pum, D.; Sleytr, U. B.; Ertl, P. Lab Chip 2013, 13, 1780-1789. doi:10.1039/c3lc41308j

8. Shin, S.-H.; Chung, S.; Sanii, B.; Comolli, L. R.; Bertozzi, C. R.; De Yoreo, J. J. Proc. Natl. Acad. Sci. U. S. A. 2012, 109, 12968-12973. doi:10.1073/pnas.1201504109 
9. Suhr, M.; Lederer, F. L.; Günther, T. J.; Raff, J.; Pollmann, K. PLoS One 2016, 11, e0156785. doi:10.1371/journal.pone.0156785

10. Chung, S.; Shin, S.-H.; Bertozzi, C. R.; De Yoreo, J. J. Proc. Natl. Acad. Sci. U. S. A. 2010, 107, 16536-16541. doi:10.1073/pnas.1008280107

11. Delcea, M.; Krastev, R.; Gutberlet, T.; Pum, D.; Sleytr, U. B.; Toca-Herrera, J. L. Soft Matter 2008, 4, 1414-1421. doi:10.1039/b719408k

12. Moreno-Flores, S.; Kasry, A.; Butt, H.-J.; Vavilala, C.; Schmittel, M.; Pum, D.; Sleytr, U. B.; Toca-Herrera, J. L. Angew. Chem., Int. Ed. 2008, 47, 4707-4710. doi:10.1002/anie.200800151

13. Toca-Herrera, J. L.; Krastev, R.; Bosio, V.; Küpcü, S.; Pum, D.; Fery, A.; Sára, M.; Sleytr, U. B. Small 2005, 1, 339-348. doi:10.1002/smll.200400035

14. Wetzer, B.; Pum, D.; Sleytr, U. B. J. Struct. Biol. 1997, 119, 123-128. doi:10.1006/jsbi.1997.3867

15. Moreno-Cencerrado, A.; Iturri, J.; Pum, D.; Sleytr, U. B.; Toca-Herrera, J. L. Polymer 2016, 102, 379-385. doi:10.1016/j.polymer.2016.03.039

16. Sherratt, M. J.; Holmes, D. F.; Shuttleworth, C. A.; Kieltyy, C. M. Biophys. J. 2004, 86, 3211-3222. doi:10.1016/S0006-3495(04)74369-6

17. Lopez, A. E.; Moreno-Flores, S.; Pum, D.; Sleytr, U. B.; Toca-Herrera, J. L. Small 2010, 6, 396-403. doi:10.1002/smll.200901169

18. López, A. E.; Pum, D.; Sleytr, U. B.; Toca-Herrera, J. L. Phys. Chem. Chem. Phys. 2011, 13, 11905-11913. doi:10.1039/c1cp00052g

19. Frohnmayer, J. P.; Brüggemann, D.; Eberhard, C.; Neubauer, S.; Mollenhauer, C.; Boehm, H.; Kessler, H.; Geiger, B.; Spatz, J. P. Angew. Chem., Int. Ed. 2015, 54, 12472-12478. doi:10.1002/anie.201503184

20. Iturri, J.; García-Fernández, L.; Reuning, U.; García, A. J.; del Campo, A.; Salierno, M. J. Sci. Rep. 2015, 5, 9533. doi:10.1038/srep09533

21. Molino, P. J.; Higgins, M. J.; Innis, P. C.; Kapsa, R. M. I.; Wallace, G. G. Langmuir 2012, 28, 8433-8445. doi:10.1021/la300692y

22. Rodahl, M.; Höök, F.; Fredriksson, C.; Keller, C. A.; Krozer, A.; Brzezinski, P.; Voinova, M.; Kasemo, B. Faraday Discuss. 1997, 107, 229-246. doi:10.1039/a703137h

23. Tharad, S.; Iturri, J.; Moreno-Cencerrado, A.; Mittendorfer, M.; Promdonkoy, B.; Krittanai, C.; Toca-Herrera, J. L. Langmuir 2015, 31, 10477-10483. doi:10.1021/acs.langmuir.5b02849

24. Ilk, N.; Kosma, P.; Puchberger, M.; Egelseer, E. M.; Mayer, H. F.; Sleytr, U. B.; Sára, M. J. Bacteriol. 1999, 181, 7643-7646.

25. Sára, M.; Dekitsch, C.; Mayer, H. F.; Egelseer, E. M.; Sleytr, U. B. J. Bacteriol. 1998, 180, 4146-4153.

26. Sleytr, U. B.; Sára, M.; Küpcü, Z.; Messner, P. Arch. Microbiol. 1986, 146, 19-24. doi:10.1007/BF00690152

\section{License and Terms}

This is an Open Access article under the terms of the Creative Commons Attribution License (http://creativecommons.org/licenses/by/4.0), which permits unrestricted use, distribution, and reproduction in any medium, provided the original work is properly cited.

The license is subject to the Beilstein Journal of Nanotechnology terms and conditions: (http://www.beilstein-journals.org/bjnano)

The definitive version of this article is the electronic one which can be found at:

doi:10.3762/bjnano.8.10 